\title{
Using Remote Lab for Enhancing E-Learning on FPAAs
}

\author{
http://dx.doi.org/10.3991/ijoe.v12i04.5230 \\ M. Felgueiras ${ }^{1}$, D. Areias ${ }^{1}$, A. Fidalgo ${ }^{1}$, C. Petry ${ }^{2}$ and G. Alves ${ }^{1}$ \\ ${ }^{1}$ Polytechnic Institute of Porto, Porto, Portugal \\ ${ }^{2}$ Instituto Federal de Santa Catarina (IFSC), Santa Catarina, Brasil
}

\begin{abstract}
Analog and digital electronic subjects are part of the electronic engineer degree but its taught is not easy because they are founded in opposite methodologies. The electronic design in the digital field is centered in the use of microprocessor and FPGA based circuits using high level programing/configuring languages. The counterpart analog design is traditionally based in the use of elementary components associated with macroblocks such operational amplifiers in order to built-up the wanted mission circuit. Some few components, as the FPAA, are analogically configurable in a similar manner already used with the FPGA. However the use of this kind of components is not straightforward once is necessary acquire some concepts not taught in the traditionally analog electronic classes.
\end{abstract}

The current work present an innovative remote lab to support teaching of the FPAAs.

Index Terms-Remote labs, FPAA, electronic design.

\section{INTRODUCTION}

The function of the engineering profession is to manipulate materials, energy and information [1]. Teaching the several subjects includes information about theory that must be complemented by the experimentation in order to build the knowledge. This completion can be a demonstration for something learned or for verifying if some experiment meets the specifications. Laboratories are essential to demonstrate laws or concepts related to a given subject [2]. In the past, traditional laboratories classes were based in lab rooms oriented by a teacher. In the last years the Remote Labs rising in importance by several reasons and are now seen as one unavoidable alternative in practical teaching [3].

Electronic teaching includes the digital and analog but the associated Electronic Design strategies currently used in these two arenas are completely opposite. In fact, the first is based in the use of programs/configuration using a standard languages, in a hardware changing-less approach. By the contrary, the second one is traditionally based in the use of typical circuit topologies adapted to each situation in where each change is made by hardware modification.

Some few components such the Field Programmable Analog Arrays (FPAA) [4, 5] or the Programmable System-on-Chip (PSoC) [6] allows to make the analog electronic design in a manner similar to the currently used in digital circuits, through a program/configuration. Differentially than the digital counterpart, the use of this components is not straight once involves the knowledge of some particular concepts.
This work present remote lab support the e-Learning of FPAA.

\section{EleCtronic TEACHING}

Teaching is structured activity that encompasses several issues such as the reuse earlier concepts to transmit new ones. One major limitation is that the several subjects to teach remains in a significant different state of development of its components and for so imposing different approach design methodologies. This is the case of teaching electronics subjects where the digital electronic arena present a very high development state when compared by the analog electronic arena.

In fact the first arena, the digital electronic, is based in the use of flexible circuits that at present are based in microprocessor (e.g. microcontrollers) or Field Programmable Gate Array (FPGA). The development of a Mission Circuit is often made by using normalized programing languages (e.g. C language) for microprocessors based circuits or Hardware Description Languages (HDL) (e.g. Verilog, VHDL) for FPGA. This design methodology is based in the use of a relatively high level set of instructions. On this procedure, the user start from an initial state where they are e set of requirements and then design a high level solution often under a form of a chart, graph, etc. After this solutions is translated for the kind of flexible circuit chosen, i.e. for microcontroller or FPGA. If used a microprocessor based circuit is common the appropriate assembler language or another synthesizable language (e.g. C language). If used a FPGA is possible the use of a HDL proprietary language or, more commonly, the use of a standard language such the VHDL or Verilog. In any case, the solution is developed having in a base a set of instructions that is organized in order to produce an intended Mission Circuit.

In the second arena, the analog electronic, is traditionally based in the use of elementary components (e.g. resistors, capacitors, diodes, transistors, etc.) or macroblocks (e.g. Operational Amplifiers). The user must know how each component works and need to associate then in order to obtain the intended the Mission Circuit. Is often the use of the so called cookbook, i.e. a handbook full of a long set of generic circuits that can be configurable for a given application. We can allocate the several analog components into two levels: the most elementary level and the macroblocks level. The first includes elementary components such resistors, capacitors, diodes and so on. The second includes blocs such Operational Amplifiers, Programmable Gain Amplifiers, filters and so on. In this last the user frequently only have knowledge at pins interface 
level. To design a mission circuit, the designer starts from the most elementary level (e.g. resistor, capacitors), associated and the next level (e.g. operational amplifier) in order to produce an intended solution that belongs to the third level. This design methodology is very hard once starts repeatedly from the most elementary level and corresponds to constant reinvention is the wheel. These approaches are depicted in the figure 1.

As seen, the digital arena uses a top-down design methodology whereas in the analog arena uses a bottom-up design methodology.

Those two main approaches carry some difficulties for both students and teachers. Being opposite, this often bring some difficulties.

First of all they are confusing. In fact those two methodologies are opposite, and the student chooses to have one only. He prefers to remain only in the digital arena, once is more high level and thus more close to the human way to think.

Secondly, those methodologies are different when a circuit changing mode is required during the circuit debug phase. This step is part of the developing circuit process and includes several debugging steps once none intended mission circuit works correctly at first time. Debug operations takes always place, no matter if it is analog or digital. However, in the digital all corrections are made at program / configuration level in a hardware changing-less manner. By contrary, in the analog arena all changes includes some degree of hardware change, with the all difficulties associated. Experience shows that the first methodology is very appealing to the new generation of students.

Finally the components updating process is different in each arena. The first methodology takes advantage from the new components characteristics once we just use programming languages. In this way, the user can take advantage from the regular improvements in components features such new powerful instructions, speed, size etc. The second one is not so straight. Electronic manufactures put continually new components in the market and the analog designer needs to expend time for its own personal updating.

From the previously exposed is clear that the first methodology is the most attractive one if feasible for both electrical arenas. They are some few analog components such the PSoC and the FPAA. Although its interesting features, they are not often considered in the engineering school curricula.

\section{Analog CONFigurable COMPONENTS}

The FPAA is an analog configurable component that allows to be configured using the very same methodology used to the digital flexible circuits. The figure 2 presents the FPAA development kit.

However, the use of this components is not straightforward once present some drawbacks that should be taken in account to be possible the transition between the traditional manner of teaching analog design to the one using FPAA. It is necessary to establish some steps to make the use of the FPAA acceptable by the traditional student that comprehends the development of:

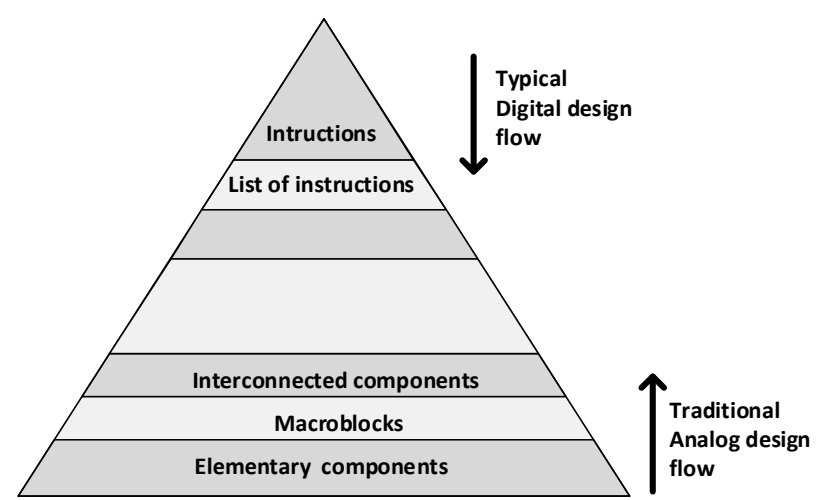

Lower level

Figure 1. Analog and digital methodologies design flow.

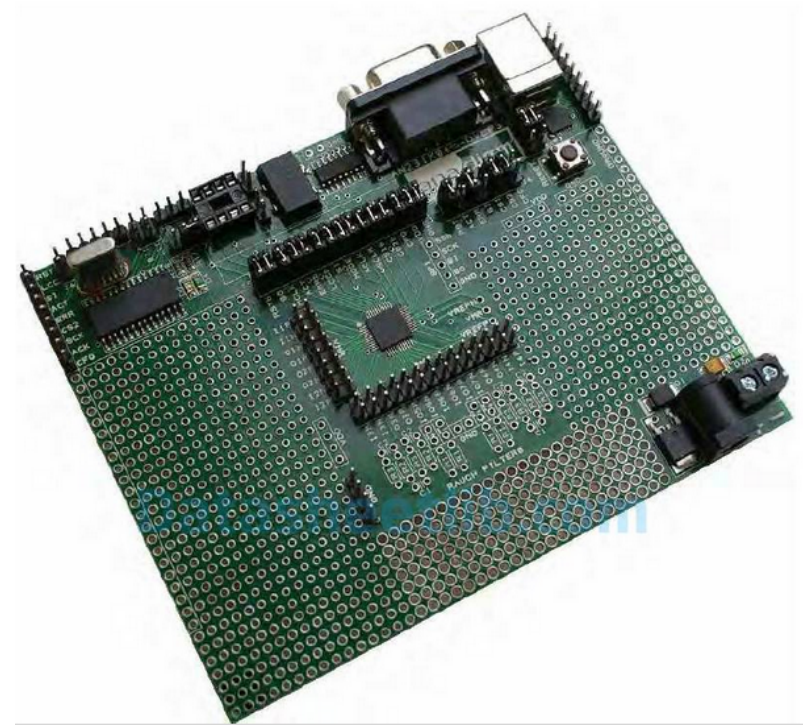

Figure 2. The FPAA development kit.

- A set of means to clarify concepts

- A remote lab to practice with FPAA

- A set of means to inform the student about his knowledge level

The first includes the development of writing notes, slides, flash movies, etc., to explain some notions related to analog design such ground (e.g. differences between ground and earth, power supply ground, analog ground, digital ground etc.), types of signals (digital, analog, quasi-digital), signals references (single-ended signal, doubleended signal), and switched-capacitor technology. All these concepts are important because they are intrinsically involved with the FPAA. For instance, it works with single supply and the analog ground is referred to a different voltage from the main power supply (e.g. analog ground $=1.5 \mathrm{~V}$ ). This part of the work is practically concluded and will be soon available to the community.

The second one is important to practice with FPAA without having need to buy a development kit. This point will be clarified in a subsequence section.

The third one is related to the means that will be developed to help the student to verify if his skill level match the intended and will be based in the use of the MOODLE. 


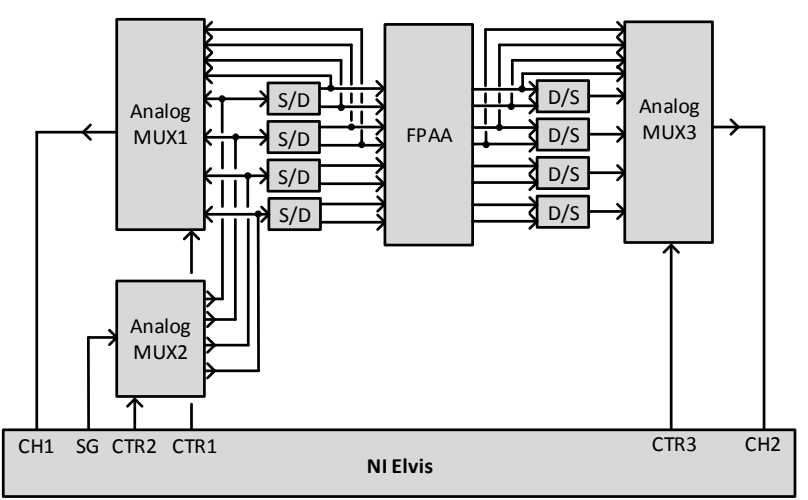

Figure 3. The FPAA remote lab infrastructure.

\section{THE FPAA REMOTE LAB}

The developed FPAA remote lab intends to provide a simple platform to remotely configuring the FPAA and verify if the actual Mission Circuit matches the intended one. This platform includes several blocks as depicted in figure 3 .

The core of all circuit is the FPAA. Its input lines are preceded by the circuit $\mathrm{S} / \mathrm{D}$ that converts single-ended signals into dual-ended signals. In the output we have the $\mathrm{D} / \mathrm{S}$ circuits that have the reverse function of the $\mathrm{S} / \mathrm{D}$, i.e., converts dual-ended signals into single-ended signals. The NI Elvis station is used to supply test signals from its internal Signal Generator (SD), to observe the input and output signal to/from the FPAA and also to control the several analog multiplexers. The MUX2 select which FPAA input will be connected to the SG. The MUX1 and MUX3 selects the FPAA input and output that will be connected to the channel one $(\mathrm{CH} 1)$ and channel two (CH2) of the internal NI ELVIS oscilloscope.

The presented platform allows to develop a significant number of experimental tests in the FPAA.

\section{CONCLUSION}

Teaching analog electronic design subjects involves a methodology other than the used in the digital electronic design mainly caused by the different state of development of the electronic components from each arena. In the digital is currently used the top-down approach whereas the bottom-up into the analog. The first is easier to use not only because is more close to the human brain but also is hardware changing-less. This work present a remote lab to support learning in FPAA. After having attain some basics of FPAA, the user will be able to verify if the developed configuration matches the intended one.

\section{REFERENCES}

[1] L. Feisel, A. Rosa, "The role of the Laboratory in the Undergraduate Engineering Education", Journal of Engineering Education, pp. 122-130, January, 2005. http://dx.doi.org/10.1002/j.21689830.2005.tb00833.x

[2] R. Silva, R. Moreira, R. Gouveia, M. Felgueiras, "Unblinding a Braille machine - A case study of reverse problem-based learning". Proceedings of the 2013 1st International Conference of the Portuguese Society for Engineering Education (CISPEE). October 31th -November 01 ${ }^{\text {st }}$, Porto, 2013. http://dx.doi.org/10.1109/ CISPEE.2013.6701968

[3] G. R. Alves, M. A. Marques, C. Viegas, M. C. Costa Lobo, "Using VISIR in a large undergraduate course: Preliminary assessment results", Proceedings of Global Engineering Education Conference (EDUCON), 2011. http://dx.doi.org/10.1109/educon.2011.57732 88

[4] J. Becker, S. Trendelenburg, F. Henrici, Y. Manoli, "A field programmable Gm-C filter array (FPAA)" Proceedings of the Second NASA/ESA Conference on Adaptive Hardware and Systems (AHS), 2007. http://dx.doi.org/10.1109/AHS.2007.3

[5] Anadigm, http://www.anadigm.com/, Visited in January 2015.

[6] Cypress - Programmable System-on-Chip, http://www.cypress. com/psoc/?source=CY-ENG-HEADER, Visited in January 2015 .

\section{AUTHORS}

M. Felgueiras, A. Fidalgo and G. Alves are teachers with Department of Electrical Engineering, School of Engineering (ISEP), Polytechnic Institute of Porto (IPP), Porto, Portugal and researchers with CIET. They research common interests include design for debug and test of mixed-signals, remote experimentation in e-learning (mcf@isep.ipp.pt, anf@isep.ipp.pt, gca@isep.ipp.pt).

D. Areias is currently perusing the MSc degree in electrical and computer engineering from School of Engineering (ISEP), Polytechnic Institute of Porto (IPP), Porto, Portugal (1120327@isep.ipp.pt).

C. Petry is teacher and researcher with the Electronic Department of the Santa Catarina Federal Institute (IFSC), Santa Catarina, Brazil. His research interests include power electronic, renewable energy and engineering e-learning platforms design (petry@ifsc.edu.br).

Submitted 09 November 2015. Published as resubmitted by the authors 14 January 2016. 